\title{
Short-term memory as a response preparation state
}

\author{
STUART T. KLAPP \\ California State University, Hayward, California 94542
}

\begin{abstract}
This review considers recent findings on motor-response programming which indicate that there is a temporary mode of storage for response commands. This storage, which is distinct from long-term memory, is required for emission of a programmed response even when there are no explicit requirements for memory. Although this system is considered to be primarily a stage in response control rather than a memory system, it may mediate verbal short-term memory when encoded in articulatory form. The implications of this perspective on short-term memory are considered.
\end{abstract}

Short-term memory (STM) is usually considered to be a limited-capacity memory from which recall occurs without retrieval or other processing. Since the concept of STM is vague, it is reasonable to suppose that more than one process may underlie the various experiments done under the title of short-term memory. The present analysis deals with one possible process, the retention of a motor program. The discussion turns first to recent research on the control of motor responses, including the articulatory response. This research has led to the concept of a state of temporary storage of response-command programs which is involved in response generation even when there is no explicit memory requirement. The possibility that much of verbal STM, as studied in the usual STM paradigms, may be mediated by the same mechanism as has been identified in these studies of motor control will then be considered. This review is intended to be interdisciplinary in the sense that it attempts to apply findings from the study of motor control to a theory of STM, thus bridging a gap between apparently unrelated fields.

\section{MOTOR PROGRAMS AND PROGRAMMING}

This section discusses the control of motor responses, including the notion of a motor program, and reaction time effects attributable to programming.

\section{Programmed Control}

The concept of a motor program refers to a means

Much of the author's research reviewed here was supported by the Small Grants Program of the Research Foundation of California State University, Hayward. The final version of this paper has benefited from comments on earlier versions from Anthony Greenwald, David Tieman, and Delos Wickens, although the author doubts that any of these reviewers would care to endorse the final theoretical statement in all of its details. Requests for reprints may be sent to Stuart T. Klapp, Department of Psychology, California State University, Hayward, California 94542. of control of muscles in which the neural commands are predetermined rather than altered as the movement progresses. Thus, there is "a set of muscle commands that are structured before a movement sequence begins" (Keele, 1968). A necessary condition for this definition to apply is the absence of feedback control, and evidence for this criterion has been emphasized by Keele (see Keele, 1968; Keele \& Summers, 1976). The contrast between feedback and programmed control has been applied successfully to demonstrate feedback control for longer duration movements and programmed control for short movements which may not allow enough time for the feedback to operate (e.g., Klapp, 1975; Schmidt \& Russell, 1972).

This method of definition, however, is not without its problems. A movement which may appear to be programmed at one level of analysis may be under feedback control at another level of analysis. An analogy may make this situation clear. Consider the control of a heating system in a building in which the thermostat is programmed for high temperature during the working day and low temperature at night. This programmed temperature change is not itself under feedback control, although feedback is involved in the actual control of the furnace, in that the actual temperature is compared to the programmed goal and the result is employed in a feedback control system to activate the furnace itself. Thus, the heating system is programmed at one level of analysis, while under feedback control at another. An analogous situation undoubtedly occurs in muscular control. Although the goal of a muscular pattern may be programmed, the execution of this program may depend on feedback based on, for example, the gamma-efferent or spindle-receptor system (see Keele \& Summers, 1976, for a description).

To take another example of a system which employs programmed control at one level of analysis and feed. back at another, consider saccadic eye movements which are initiated by a visual target and which have an extent and direction such that the target appears at the fovea at the conclusion of the movement. The 
movement, once initiated, is not altered on the basis of changes in the environmental position of the target (Westheimer, 1954), although it can be altered by internal feedback from the vestibular system (Bizzi, 1974). Thus, although saccades are programmed at an external level of analysis, there is also an element of internal feedback control. ${ }^{1}$

Another problem in using the term "programmed" to describe movement control arises when fairly long movement sequences are considered. Such sequences may be comprised of segments, each of which is programmed at the level of analysis under consideration. However, the segments may be programmed in such a way that the program for one segment is determined on the basis of feedback from execution of the preceding segment. In terms of our heating system analogy, the temperature program may be altered from time to time based on feedback from employee complaints or fuel supplier's bills. An analysis of aimed movements based on the notion that these movements are comprised of a series of separately programmed segments, with feedback used to determine the separate programs, has been presented by Keele (1968). The concept of segmental programs also appears in the analysis of sequences for which the program of the succeeding segment is not contingent on the previous segment (Klapp \& Wyatt, 1976).

The present analysis takes a different approach to the assessment of programmed control. Rather than focusing on the lack-of-feedback criterion, it returns to the original idea of structuring muscle commands prior to the onset of the movement and considers the possibility that such structuring may take measurable time. When the predicted time effects are observed, programming is assumed to have occurred. Of course, the presence of such programming does not deny the possibility of feedback control at another level. Thus, feedback may operate at a higher level, in determining which program is to be used, or at a lower level, in executing the programmed goal. The next sections describe reaction time effects attributable to programming.

\section{Choice Reaction Time and Programming}

When a response has been programmed, the neural command sequence has been organized prior to the start of the response. The time required for programming can be investigated in experiments which measure reaction time prior to the beginning of the response as a function of the nature of the response to be made. Since this research deals with programming rather than response selection, the number of alternative responses must be held constant. A relationship between reaction time and response parameters is interpreted as reflecting differences in programming times attributable to changes in the response.

Consider first the choice reaction time paradigm in which the signal marking the beginning of the reaction time interval also informs the subject which of a number of possible alternative responses is required on that particular trial. The following instances have been reported in which variation of the nature of the response leads to changes in choice reaction time:

1. Choice reaction time for pronunciation increases with the number of syllables to be pronounced. This relationship has been observed for printed or pictorial representations of words of matched frequency (Klapp, Anderson, \& Berrian, 1973) and for two-digit numbers (Eriksen, Pollack, \& Montague, 1970; Klapp, 1974). The effect is attributable to programming the response and not to perception of the visual stimulus, since the time required for perception can be independent of syllables when pronunciation is not required (Johnson, 1975; Klapp, et al., 1973). ${ }^{2}$

2. Choice reaction time is longer prior to a keypress representing the Morse code "dah" rather than the shorter "dit" (Klapp, Wyatt, \& Lingo, 1974).

3 . Choice reaction time increases with the required accuracy of short movements directed toward a target (Klapp, 1975). The restriction of this relationship to short, and apparently programmed, movements is consistent with the interpretation that such changes in reaction time are attributable to programming. According to this analysis, only the first segment of longer movements is programmed, so that reaction time is independent of the size of the target at the termination of long movements.

4. Choice reaction time increases with the physical length of movements when only minimal accuracy is required (Glencross, 1973; Klapp, 1975). Apparently the response is programmed in advance when little accuracy is required. With increased demands for accuracy, feedback control may be used to achieve greater control over the termination of the movement. For accurate movements reaction time no longer increases with length (Klapp, 1975), suggesting that long accurate movements are not totally programmed before they start.

5. Choice reaction time increases with the required movement time for moving a handle across a constant distance to a mechanical stop (Klapp \& Erwin, 1976). This suggests that changing the response duration may be a sufficient condition for observing differences in programming time.

6. Choice reaction time increases when a buttonpress is incorporated within a handle movement of fixed temporal duration and distance (Klapp \& Erwin, 1976). This indicates that changing the response duration is not a necessary condition for observing differences in programming time, even though it may be a sufficient condition.

When a relationship between choice reaction time and response parameters is observed, one can infer programmed control because the time differences are assumed to reflect programming time. However, it is not possible at present to predict when such effects 
will be observed. No effect of parameters of late segments of a response would be expected unless the entire response sequence is programmed in advance (Klapp, 1975; Klapp \& Wyatt, 1976). Although we cannot at the present state of knowledge predict when the response will be entirely programmed, the implications to be drawn in the present analysis do not rest upon the resolution of this particular issue. We can restrict our focus to cases in which choice reaction time does depend on the response parameters being considered. For such cases we next consider simple reaction time data. We then draw our implications from the contrast between choice and simple reaction time effects.

\section{Simple Reaction Time}

Although choice reaction time depends upon the nature of the programmed response which follows, simple reaction time does not necessarily show this relationship. In the choice reaction time paradigm, the signal which marks the beginning of the reaction time interval also tells the subject which response is required. By contrast, in the simple reaction time paradigm, the subject is informed in advance which particular response is to be made on the trial. Then, after a suitable time interval, a signal is given indicating that the subject is to respond as quickly as possible. Simple reaction time, measured from this second signal until the response begins, can be independent of response parameters which influence choice reaction time, provided subjects are sufficiently motivated to take advantage of the advance information. For example, the effect of syllables on pronunciation latency appeared for choice but not for simple reaction time (Klapp, 1971, 1974; Klapp et al., 1973). Similarly, for Morse code responses the longer reaction time prior to a "dah" appeared for choice reaction time but not for simple reaction time under motivating instructions (Klapp et al., 1974, Experiment 2). However, when motivating instructions were not employed, simple reaction time was independent of the response only for subjects who had short mean reaction times. These subjects can be assumed to have been self-motivated (Klapp et al., 1974, Experiment 1).

The interpretation of these findings for simple reaction time is that the subject can, but does not have to, take advantage of the advance information concerning which response to make. If the subject does utilize this information, he programs the response in advance. When the programming is done in advance of the reaction time interval, the interval is short overall and unrelated to the nature of the response to be made.

\section{TEMPORARY STORAGE OF PROGRAMS}

The contrast between choice and simple reaction time effects will now be used to argue that there must be temporary storage of muscular commands for programmed responses, a conclusion of fundamental importance for the arguments to follow. The basic data to consider are the findings that, in cases in which choice reaction time depends on the nature of the response, simple reaction time does not show this effect. The choice reaction time effect is assumed to represent differences between the programming times of the responses under consideration. Since these responses must have been in long-term memory (LTM) all along (e.g., speech is highly overlearned), programming may be regarded as conversion of the LTM representation into some other form, a form which can control the muscles. In the simple reaction time paradigm, this process must have been done in advance of the signal to respond, since the reaction time effects do not occur. Furthermore, it must be possible to hold the programmed representation until the response signal occurs. We will consider the possibility that the temporary storage required by this analysis may mediate STM as it is classically investigated.

A possible neurological interpretation of the processes identified in this analysis could assume that timed response commands are generated by long neural circuits involving multiple synapses and long pathways, and possibly involving loops between the cerebral cortex and the cerebellum (e.g., Ruch, 1965). The delays introduced in neural transmission through such circuits could generate corresponding delays in a timed sequence of motor command signals. Such neural circuits could represent the temporary storage of the above analysis. Although a few such circuits might be retained until needed, this mode of representation would appear to be inefficient for LTM because a great deal of neural tissue is required. It is reasonable to suppose that a more compact biochemical code underlies the LTM of a response sequence. Prior to generation of commands to the muscles, the temporary timing network must be established using the coded representation in LTM as a template (or "wiring diagram"), and this process leads to the choice reaction time effects.

Regardless of whether this "neurologizing" is correct, the above analysis indicates that there must be a temporary mode of storage for response commands from which a response may be generated without repeating the time-consuming programming process. While this is always involved in response control, its existence is inferred from the simple reaction time paradigm where the commands are held until the signal. Although the primary function of this temporary storage is to permit generation of motor responses, it is possible that this storage may also be involved in many experiments on STM.

\section{STM AS A MOTOR PREPARATION STATE}

This section attempts to relate the above concept of temporary storage to STM as it is more traditionally studied. 


\section{General Considerations}

In the typical verbal STM experiment, words (or letters or numbers) are presented to the subject. After a brief retention interval, the items are to be recalled or recognized. As Tulving (1972) has observed, the subject knows the items and their meaning all along (semantic memory), so in a sense it is not reasonable to say that he "learns" them during the experiment. What the subject does learn is that a particular item has occurred on a particular experimental trial, that is, in a particular episode of his life. Tulving introduced the term "episodic memory" to describe this function. In the case of short-term episodic memory, the subject could keep track of which items were presented on a given trial by programming the required articulatory response in advance and holding the program in the state of temporary storage which was identified in the above analysis. At the end of the retention interval the subject could emit whatever speech responses happened to be programmed at that time. Thus, in principle, verbal STM could be mediated by temporary storage of articulatory response programs.

The hypothesis that verbal STM is mediated by the temporary representation of the articulatory response sequence appears to reflect the intuitive properties usually ascribed to STM. First, STM is usually viewed as having limited capacity, and this is true also of the above concept of the temporary storage. Second, the memory items in STM are usually assumed to be immediately available, so that retrieval is not required. This property also follows directly from the above concept of the temporary state, from which a response can be issued without the need for the programming required when only the LTM representation is available.

The process by which material is retained in STM has frequently been called "rehearsal." However, it is quite unlikely that any overt articulation is required, since suppression of muscular activity by feedback does not reduce STM performance (Glassman, 1972). The response nature of STM is retained in the present theory, while involvement of overt responses is seen as unnecessary since STM is mediated by a response program rather than by a response. The overt articulation may occur, but this is not necessary for STM retention.

\section{Articulatory Coding in Verbal STM}

The above analysis of verbal STM can only apply when the material is retained in terms of the articulatory response required at output. The assumption that some, and possibly most, situations which have been identified as verbal STM involve articulatory coding seems consistent with the data. Conrad (1964) first demonstrated that, with visual presentation and written recall, intrusion errors in verbal STM tend to be items which sound like the correct item. Since words which sound alike are also articulated alike, this finding could imply an underlying memory system in either auditory or articulatory form. Attempts to decide between these alternative interpretations of the nature of intrusion errors have not settled the matter (Hintzman, 1967; Wickelgren, 1969).

A more direct approach to the study of encoding modality is to prevent articulatory coding by requiring the subject to articulate an irrelevant sound during presentation of the test item. Levy (1971) reported that this procedure can reduce recall in a probed recall paradigm to the chance level, provided input is visual without accompanying audition. When auditory information is also provided, however, a reasonable level of recall is obtained even in the presence of irrelevant articulation. It would appear reasonable to suppose that verbal STM can be mediated by articulation, as the present analysis suggests, although it should be clear that other modalities of representation may also be possible. This review does not consider all possible STM phenomena, but is limited to phenomena associated with articulatory and other motor representations.

\section{Rate of Input into STM}

According to the present analysis, response programming leads to the formation of articulatory STM. The rate of input into STM should equal the rate of programming if the two processes are identical. However, these two ways of considering the same process suggest different operations for measurement.

The rate of programming for articulation, in time per syllable, can be assessed by the change in choice reaction time as the number of syllables to be pronounced is increased. However, a complication is introduced by the finding that choice reaction time seems to increase less per syllable when the total number of syllables is larger (Klapp \& Wyatt, 1976, Table 1). This could mean that longer sequences of speech are not entirely programmed prior to the beginning of the response, so that only the first few syllables of the response are programmed during the reaction time interval. Programming of the remainder of the response may be postponed until later intervals. In support of the notion that only the initial segments of a long response are preprogrammed, Klapp and Wyatt (1976) reported that, for sequences of two Morse code responses, programming of only the initial dit or dah is reflected in the reaction time. Differences in the length of the interresponse interval as a function of the second dit or dah suggest that programming of the second response segment occurs during this interval, rather than before the response begins. If this analysis is valid, then the best estimate of the time required to program a syllable of speech can be obtained from the effects of adding one syllable to the shortest verbal response. Short speech sequences may be programmed entirely before they start, while only part of longer sequences are preprogrammed. For comparisons between pronunciation of one- and two-syllable words, Klapp et al. (1973) reported differences in choice reaction time of 
$14 \mathrm{msec}$. The effect becomes smaller for longer sequences of speech, for example, $6.6 \mathrm{msec}$ for the comparison between three- and four- syllable numbers (Klapp, 1974).

A different operation has been used by Sperling (1963) to measure the rate of input into STM. Sets of letters were presented visually. After a variable interval, this presentation was followed by a mask comprised of visual noise. It is assumed that the mask immediately interrupted further processing of the stimulus or its iconic representation, although this assumption has been questioned (Eriksen \& Eriksen, 1971). After the presentation of the stimulus and mask, the subject was to immediately recall as many letters as possible. Within the assumptions of this analysis, the slope of the function relating letters recalled to mask delay, which is $10 \mathrm{msec} / \mathrm{letter}$, is an estimate of the rate at which items are encoded into STM. Since letters are primarily monosyllabic, this estimate may also be considered to represent the time required to enter one syllable into STM.

Since the rate of formation of STM $(10 \mathrm{msec} / \mathrm{syllable})$ and the rate of programming of articulation $(14 \mathrm{msec} / \mathrm{syllable}$ or less) are of the same order of magnitude and probably agree within the errors of measurement, we conclude that programming and formation of STM could be the same process as the present theory asserts. Since overt speech requires about $100 \mathrm{msec} / \mathrm{syllable}$ (Sperling, 1963), response production apparently is not involved in this programming or STMformation process.

\section{Distraction Effects}

A frequent experimental example of verbal STM is the distractor paradigm, sometimes called the BrownPeterson paradigm (Brown, 1958; Peterson \& Peterson, 1959). The fundamental finding is that a few verbal items can be retained indefinitely in the absence of distraction during the retention interval. However, when distraction is present (usually counting backward by threes with overt vocalization), the probability of recall declines markedly within a few seconds. ${ }^{3}$ The distractor does not have to be articulatory-keypressing and manual-pursuit rotor tasks have similar effects (Watkins, Watkins, Craik, \& Mazuryk, 1973). According to the present theoretical framework, the distractor places excessive demands on the temporary response storage system which may be assumed to have limited capacity to hold response programs regardless of the modality of the response. Since the present theory holds that this response system is used in verbal STM, rapid forgetting would be expected when the competing response programs are introduced.

This view leads to two additional predictions: (1) Retention-interval events not involving programming of speech or other responses should not be effective distractors. (2) STM for nonverbal motor responses should be subject to distraction in a manner analogous to the effects observed for verbal memory, since memory for motor and verbal items is assumed to be mediated by the same response-programming system. Evidence bearing on these predictions is now considered.

Events which do not distract. An example of a potential distractor task which does not involve motor programming would be signal detection within a background of noise. Even when this task is made quite difficult, it does not seem to interfere with STM to as great an extent as do the response-requiring distractors (Reitman, 1971, 1974; Shiffrin, 1973). Other examples of retention-interval events which should not and do not distract verbal retention include the presentation of tones (Watkins et al., 1973) or loud speech sounds (Sperling, 1963). It appears that response programming may be a necessary condition for the occurrence of distraction effects in verbal STM, as the present thecry predicts. $^{4}$

Verbal distractors affect motor memory. The possibility that the customary verbal arithmetic distractor would produce rapid loss of STM for nonverbal motor responses has led to many experiments. However, most of these are not suitable for the present theoretical issue, since they involve having a blindfolded subject move a handle to a mechanical stop, after which he attempts to generate a movement which terminates at the same point. As Kantowitz (1974) has observed, the recall movement in this paradigm is drastically different from the input movement. The input movement is suddenly terminated by the mechanical stop, while the recall movement terminates gradually as the subject reaches the estimated end point. Experiments of this type cannot be considered to involve memory for a program of a particular movement. Another approach to the study of motor STM is to test retention by recognition rather than by recall, so that both input and output movements are terminated by a mechanical stop and are, therefore, similar (Kantowitz, 1974). However, this procedure is not useful for the present analysis either, since it involves movement to an experimenter-determined stop, rather than movement generated from a program.

An experiment which is relevant to the present issue involved allowing the subject to determine his own movement at input, and then requiring him to reproduce that same movement at the end of the retention interval (Jones, 1974). For both constant and variable error measures there was no loss of memory over a $15-\mathrm{sec}$ unfilled interval, but considerable loss over a 15-sec interval filled with the customary verbal counting distractor. It appears that STM for nonverbal motor responses is lost in the presence of distraction requiring programming, as the present theory predicts.

\section{Limit of Capacity of STM}

Although it has long been recognized that STM has only a limited capacity, determining the appropriate metric for expressing this limit has been an elusive 
problem. In his classic paper, Miller (1956) showed that the limitation of STM capacity could not be expressed in terms derived from information theory. The motor program interpretation of STM suggests why information measures are not suitable. Only a limited amount of response activity can be programmed and held in the state of preparation, and this limits the capacity of STM. The information conveyed by this limited response activity is not a relevant consideration.

The present theory suggests that responses which take longer to program may also occupy more space in temporary storage. Since programming time depends on the number of syllables for speech, the span of STM should be shorter for verbal items with more syllables. A shorter span for items of more syllables was reported by Craik (1968), although he did not emphasize this feature of his data. When the capacity of STM has been estimated by indirect measures based on free recall of lists beyond the STM span, the results have not shown any difference in the STM component as a function of the number of syllables per item (Craik, 1968; Glanzer \& Razel, 1974). Thus, span measures and free-recall measures lead to conflicting conclusions regarding the role of syllables in STM capacity. However, it would appear that the results from the more direct approach should be accepted, since fewer assumptions are required to interpret the findings. It should be emphasized that, consistent with the present theory, memory span did decrease as the number of syllables per item increased.

\section{STM WITHIN THE FLOW OF INFORMATION}

The implications of the present theory of STM for broader theories of information processing will now be considered.

\section{Contrasting Roles of STM and LTM}

The present theory assigns an entirely different character to STM and LTM. Although LTM is mediated by a system with memory as its primary function, much of STM is mediated by a system for which "memory" is a by-product of response control. This viewpoint is consistent with the findings that brain injury can lead to problems in forming new LTM representations, while normal performance is retained for STM in the absence of distraction (Baddeley \& Warrington, 1970; Milner, 1966). The present theory suggests that these findings imply that the systems associated with motor control are normal; difficulties arise when actual memory operations are attempted. The notion that STM is attributable to motor control rather than to "memory" is also consistent with the possibility that, under certain circumstances, the same material can be entered again and again into STM without any cumulative carry-over from presentation to presentation (Cohen \& Johansson, 1967).

A common view of the function of STM holds that material is encoded, rather than retained in raw sensory form. Thus, during formation of STM, some contact with LTM must have occurred to permit, for example, encoding sounds as words. In the present theory, the nature of this interaction between LTM and STM is specified to some extent. Incoming sensory material contacts the LTM representation of the motor response to be executed at recall. The LTM representation serves as the template for the programming process, and the programmed representation is held temporarily until the overt response is required.

\section{STM as a Terminal Stage}

The present view that STM is used to hold motor programs prior to release as an overt response suggests that the role of STM in information processing occurs subsequent to all steps other than the actual execution of the response. If accepted, this view would require some changes in the usual view of the role of STM. Information flow diagrams would show STM at the termination rather than at the beginning of the flow of information. The view that STM represents the working space of information processing (i.e., consciousness ${ }^{5}$ ) would be questioned, since an output storage buffer would be an unlikely candidate for this role. Somewhat more in keeping with current thinking is the prediction that, if STM operates at output rather than at input, then verbal material might be deeply processed prior to becoming lost to STM. We turn in this section to a consideration of some studies in selective attention which seem to support this possibility.

Selective attention may occur when the rate of incoming information exceeds the capacity of some portion of the information-processing system. Since STM has limited capacity, it represents one potential bottleneck in information flow at which selective attention could occur. Indeed Shiffrin and his associates (e.g., Shiffrin, Pisoni, \& Casteneda-Mendez, 1974) report no attention effects when demands on STM are low, suggesting that attentional effects observed in other contexts may be attributable to the failure of STM to handle all the information reaching it. According to the present theory, the articulatory STM system is associated with the response or output of the flow of information. Therefore, when selective attention is associated with the limits of articulatory STM, the rejected material could have been fully perceived before becoming lost.

In the shadowing paradigm the subject repeats (shadows) word for word a verbal message arriving in one ear while a second message is presented to the other ear. Recall of material from the nonshadowed ear is very poor. According to the present theory, shadowing keeps the response system occupied, thereby preventing programming of items from the nonshadowed ear. Since programming and formation of articulatory STM are equivalent, recall is poor. However, the nonshadowed material could have been perceived 
even though it failed to become programmed as a response, since the programming limitation is at output rather than input.

There is fairly substantial evidence to support this possibility. The response latency for shadowing can be influenced by the relationship of the meanings of the shadowed and nonshadowed messages even without recall of the latter (Lewis, 1970; Triesman, Squire, \& Green, 1974). The nonshadowed message can also influence interpretation of ambiguous messages in the shadowed ear, again without recall (MacKay, 1973). Somewhat more questionable are the results of Corteen and his associates (Corteen \& Dunn, 1974; Corteen \& Wood, 1972), who classically conditioned a galvanic skin response by pairing shock with city names. When city names appeared later in the nonshadowed ear, there was no recall. Nevertheless, the galvanic skin response occurred at presentation even for cities which had not been paired with shock during the conditioning phase. However, Wardlaw and Kroll (1976) report a convincing failure to replicate these conditioning results, so this particular line of evidence is not as solid as evidence from the other paradigms. Thus, there is converging evidence from two, and possibly from three, approaches which suggest that nonshadowed material is processed to a deep level of semantic analysis before it is lost. This is consistent with the present theory that STM operates late in the flow of information.

\section{CONCLUSIONS}

The study of motor-response control suggests that a programmed response cannot be controlled directly from its representation in LTM. A transformation is needed to generate a temporary storage which can control the response. Since the transformed representation can be retained until the response occurs, it has some memory aspects and may mediate the recall observed in experiments involving articulatory STM. According to this view, the primary function of the temporary storage is to provide a response buffer which is always involved, even when there is no explicit memory requirement, in the production of the response. Thus, this system may be considered to be a system of motor control rather than a true "memory" system. In a sense, therefore, the analysis holds that memory is not involved in some experiments which have traditionally been known as STM experiments. However, the possibility of other forms of STM, including a sensory memory, is not denied by this analysis.

\section{REFERENCES}

ALPERN, M. Eye movements. In Handbook of sensony physiology (Vol. VII/4). New York: Springer-Verlag, 1972.

Baddeley, A. D., \& Warrington, E. K. Amnesia and the distinction between long-and short-term memory. Journal of Verbal Learning and Verbal Behavior, 1970, 9, 176-189.

Barron, R. W., \& Pitrenger, J. B. The effect of orthographic structure and lexical meaning on "same-different" judgments.
Quarterly Journal of Experimental Psychology, 1974, 26, 566-581.

Bızzı, E. The coordination of eye-head movements. Scientific American, 1974, 231, 100-106.

Brown, J. Some tests of the decay theory of immediate memory. Quarterly Journal of Experimental Psychology, 1958, 10, 12-21.

Cohen, R. L., \& Johansson, B. S. The activity trace in immediate memory: A re-evaluation. Journal of Verbal Leaming and Verbal Behavior, 1967, 6, 139-143.

Conrad, R. Acoustic confusions in immediate memory. British Journal of Psychology, 1964, 55, 75-84.

Corteen, R. S., \& DunN, D. Shock-associated words in a nonattended message: A test for momentary awareness. Journal of Experimental Psychology, 1974, 102, 1143-1144.

Corteen, R. S., \& Wood, B. Autonomic responses to shockassociated words in an unattended channel. Journal of Experimental Psychology, 1972, 94, 308-313.

CRAIK, F. I. M. Two components in free recall. Journal of Verbal Learning and Verbal Behavior, 1968, 7, 996-1004.

ERIKSEN, C. W., \& ERIKSEN, B. A. Visual perceptual processing rates and backward and forward masking. Journal of Experimental Psychology, 1971, 89, 306-313.

Eruksen, C. W., Pollack, M. D., \& Montague, W. E. Implicit speech: Mechanism in perceptual encoding? Journal of Experimental Psychology, 1970, 84, 502-507.

Festinger, L., \& Easton, M. Inferences about the efferent system based on a perceptual illusion produced by eye movements. Psychological Review, 1974, 81, 44-58.

Forster, K. I., \& Chambers, S. M. Lexical access and naming time. Journal of Verbal Leaming and Verbal Behavior, 1973, 12, 627-635.

Friden, T. P. The effects of familiarity in a perceptual matching task. Perception \& Psychophysics, 1973, 14, 487-492.

Glanzer, M., \& Razel, M. The size of the unit in short-term storage. Joumal of Verbal Learning and Verbal Behavior, $1974,13,114-131$.

Glassman, W. E. Subvocal activity and acoustic confusions in short-term memory. Journal of Experimental Psychology, 1972, 96, 164-169.

Glencross, D. F. Response complexity and the latency of different movement patterns. Journal of Motor Behavior, 1973, 5, 95-104.

Henderson, L., Coltheart, M., \& Woodhouse, D. Failure to find a syllabic effect in number naming. Memory \& Cognition, 1973, 1, 304-306.

Hintzman, D. L. Articulatory coding in short-term memory. Journal of Verbal Learning and Verbal Behavior, 1967, 6, $312-316$

JoHNSON, N. F. On the function of letters in word identification: some data and a preliminary model. Joumal of Verbal Learming and Verbal Behavior, 1975, 14, 17-29.

JONEs, B. Role of central monitoring of efference in short-term memory for movements. Joumal of Experimental Psychology, $1974,102,37-43$.

KANTOWITZ, B. H. Modality effects in recognition short-term memory. Journal of Experimental Psychology, 1974, 103, 522-529.

KEELE, S. W. Movement control in skilled motor performance. Psychological Bulletin, 1968, 70, 387-403.

KeEle, S. W., \& Summers, J. J. The structure of motor programs. In G. E. Stelmach (Ed.), Motor control: Issues and trends. New York: Academic Press, 1976.

Keppel, G., \& Underwood, B. Proactive inhibition in shortterm retention of single items. Journal of Verbal Learning and Verbal Behavior, 1962, 1, 153-161.

KLAPP, S. T. Implicit speech inferred from response latencies in same-different decisions. Journal of Experimental Psychology, 1971, 91, 262-267.

KLAPP, S. T. Syllable-dependent pronunciation latencies in number-naming, a replication. Joumal of Experimental Psychology, 1974, 102, 1138-1140. 
KLAPP, S. T. Feedback versus motor programming in the control of aimed movements. Journal of Experimental Psychology: Human Perception and Performance, 1975, 104, 147-153.

KIAPP, S. T., ANderson, W. G., \& Berrian, R. W. Implicit speech in reading, reconsidered. Joumal of Experimental Psychology, 1973, 100, 368-374.

KiAPP, S. T., \& ERwiN, C. I. Relation between programming time and duration of the response being programmed. Journal of Experimental Psychology: Human Perception and Performance, 1976, in press.

KIAPP, S. T., \& WyatT, E. P. Motor programming within a sequence of responses. Joumal of Motor Behavior, 1976, 8, 19-26.

Kiapp, S. T., Wyatt, E. P., \& Lingo, W. M. Response programming in simple and choice reactions. Journal of Motor Behavior, 1974, 6, 263-271.

KiAtzKY, R. L. Human memory. San Francisco: W. H. Freeman, 1975.

LEVY, B. A. Role of articulation in auditory and visual short-term memory. Journal of Verbal Learning and Verbal Behavior, 1971, 10, 123-132.

LEwIs, J. L. Semantic processing of unattended messages using dichotic listening. Journal of Experimental Psychology, $1970,85,225-228$.

MACKAY, D. G. Aspects of the theory of comprehension, memory and attention. Quarterly Journal of Experimental Psychology, 1973, 25, 22-40.

MASSARo, D. W. Retroactive interference in short-term recognition memory for pitch. Joumal of Experimental Psychology, 1970, 83, 32-39.

Miller, G. A. The magical number seven, plus or minus two: Some limits on our capacity for processing information. Psychological Review, 1956, 63, 81-97.

Milner, B. Amnesia following operation on the temporal lobes. In C. W. M. Whitty, \& O. L. Zangwill (Eds.). Amnesia. London: Butterworths, 1966.

Peterson, L. R., \& Peterson, M. Short-term retention of individual items. Journal of Experimental Psychology, 1959, 58, 193-198.

Pynte, J. Readiness for pronunciation during the reading process. Perception \& Psychophysics, 1974, 16, 110-112.

Reitman, J. S. Mechanisms of forgetting in short-term memory. Cognitive Psychology, 1971, 2, 185-195.

Reitman, J. S. Without surreptitious rehearsal, information in short-term memory decays. Journal of Verbal Learning and Verbal Behavior, 1974, 13, 365-377.

Ruch, T. C. Basal ganglia and cerebellum. In T. C. Ruch, H. D. Patton, J. W. Woodbury, \& A. L. Towe Neurophysiology (2nd ed.). Philadelphia: W. B. Saunders, 1965.

SChmid, R. A., \& Russell, D. G. Movement velocity and movement time as determiners of the degree of preprogramming in simple movements. Journal of Experimental Psychology, 1972, 96, 315-320.

ShIFFru, R. M. Information persistence in short-term memory. Journal of Experimental Psychology, 1973, 100, 39-49.

Shiffrin, R. M., Pisoni, D. B., \& Castaneda-Mendez, K. Is attention shared between the ears? Cognitive Psychology, 1974, 6, 190-215.

SPERLing, G. A. A model of visual memory tasks. Human Factors, 1963, 5, 19-31.

STOPER, A. E. Apparent motion of stimuli presented stroboscopically during pursuit movements of the eye. Perception \& Psychophysics, 1973, 13, 201-211.

TATUM, B. C., \& FrIDEN, T. P. Verbal and imaginal coding of words and pictures. Perceptual and Motor Skills, 1974, 39, 551-561.

Treisman, A., Squire, R., \& Green, J. Semantic processing in dichotic listening? A replication. Memory \& Cognition, $1974,2,641-646$.

Tulving, W. Episodic and semantic memory. In E. Tulving \& W. Donaldson (Eds.), Organization of memory. New York: Academic Press, 1972.

Wardlaw, K. A., \& Kroll, N. E. A. Autonomic responses to shock-associated words in a nonattended message: A failure to replicate. Journal of Experimental Psychology: Human Perception and Performance, 1976, 2, 357-360.

Watkins, M. J., Watkuns, O. C., CRaik, F. I. M., \& MAzURYK, G. Effect of nonverbal distraction on short-term storage. Jourmal of Experimental Psychology, 1973, 101, 296-300.

Waugh, N. C., \& Norman, D. A. Primary memory Psychological Review, 1965, 72, 92-93.

Westheimer, G. Mechanism of saccadic eye movements. Archives of Ophthalmology, 1954, 52, 932-941.

WiCKelgReN, W. A. Auditory and articulatory coding in verbal short-term memory. Psychological Review, 1969, 76, 232-235.

\section{NOTES}

1. In contrast to the programmed saccade, pursuit eye movements are not planned in advance. Alpern (1972) has summarized much of the physiological evidence on the contrast between these modes of control of the same eye muscles. The conclusion that saccadic, but not pursuit, eye movements are programmed is also supported by the finding that a memory of the program is available for interpretation of movements of an image on the retina after a programmed saccade, but not after a pursuit movement for which no program has been generated (Festinger \& Easton, 1974; Stoper, 1973).

2. Some detailed comments on this phenomenon may be of interest to readers who are following this research closely. First, duration of pronunciation does not necessarily depend on number of syllables for sets of stimuli which show the syllable effect in reaction time (Klapp \& Erwin, 1976). Second, the effect of syllables does not always occur for tasks which require reading but not programming of articulation. Some studies report no such effect (Barron \& Pittenger, 1974; Johnson, 1975; Klapp et al., 1973), while others find an effect (Friden, 1973; Klapp, 1971; Pynte, 1974; Tatum \& Friden, 1974). It is perhaps best to continue to hold (as in Klapp et al., 1973) that syllable effects for stimulus recognition represent an optional and hence unstable process, while the corresponding effect for pronunciation represents an obligatory process of programming the articulation. A third comment concerns the replicability of the syllable effect for pronunciation. A failure to replicate for number stimuli (Henderson, Coltheart, \& Woodhouse, 1973) can be attributed to methodological problems (see Klapp, 1974) and to the smaller change in reaction time, per syllable, for stimuli which are longer overall (see Klapp \& Wyatt, 1976, Table 1). Forester and Chambers (1973) report only a small, nonsignificant effect of syllables on pronunciation latency in data averaged across words and nonwords. However, when only easily recognized words are considered (see their appendix), a large difference in latency as a function of syllables appears. Unfortunately, the authors do not report statistics for this comparison.

3. Recall decrements attributable to the distractor do not appear on the first few trials of an experiment (Keppel \& Underwood, 1962). Apparently a system is available for retention which does not share processing space with the distractor task, but which becomes useless in the presence of proactive interference. The present discussion is restricted to the processes which can be employed in the presence of substantial proactive interference. 
4. By contrast to the findings for retention of verbal material, sounds during the retention interval do interfere with retention of a single abstract tone (Massaro, 1970). For verbal materials, the present theory claims, STM is in motor form so that only motor events interfere. For a single tone which cannot be represented as a response, a sensory mode of storage which is susceptible to disruption by new auditory input may be used.

5. Klatsky (1975, Chapter 5) traces this view to Sigmund
Freud, while Waugh and Norman (1965) trace it to William James. However, it is not clear that these classical concepts correspond to STM as currently investigated by experimental psychologists.

(Received for publication December 3, 1975; revision accepted April 26, 1976.) 